\title{
10 Kostensensible Leitlinien: Einschätzungen von Patienten
}

\author{
Swantje Reimann und Georg Marckmann
}

\subsection{Hintergrund und Ziele der Studie ${ }^{146}$}

Die Kostensensiblen Leitlinien (KSLL) wurden insbesondere auch unter der Annahme entwickelt, dass diese Form der expliziten Leistungssteuerung nicht nur medizinisch und ökonomisch rationaler ist, sondern vor allem auch für die betroffenen Patienten eher akzeptabel: Alle Patienten (zumindest im Bereich der GKV) erhalten nach den gleichen expliziten Vorgaben auf der Grundlage ethisch gut begründeter Kriterien Zugang zu innovativen medizinischen Maßnahmen. Die Entscheidung muss nicht im Einzelfall durch den behandelnden Arzt gefällt werden, was die Arzt-Patient-Beziehung entlasten könnte. Ob diese Annahmen tatsächlich zutreffen, ließe sich eigentlich wiederum nur mit einer Interventionsstudie ermitteln, bei der die KSLL eingesetzt und die betroffenen Patienten nach ihrer Einschätzung dazu befragt werden. Da eine solche Interventionsstudie aus sozialrechtlichen Gründen im System der GKV nicht durchführbar ist, entschieden wir uns hilfsweise für eine Befragung von Patienten, die aufgrund ihrer Erkrankung potenziell von den KSLL betroffen sein könnten. Dabei verfolgten wir die übergreifende Zielsetzung, eine Einschätzung der Stärken und Schwächen sowie der Akzeptanz expliziter Leis- 
tungsbegrenzungen mittels KSLL aus Sicht der Betroffenen zu gewinnen. Als Gesprächsgrundlage diente eine für medizinische Laien angepasste Version der KSLL zu Medikamente-freisetzenden Stents (drug eluting stents, DES), zusammen mit einer allgemeinverständlichen Einführung in das Modell der KSLL. Nach unserem Wissen handelt es sich um die erste empirische Untersuchung der Einschätzung von Patienten zu expliziten Instrumente der Leistungssteuerung im deutschen Gesundheitswesen.

\subsection{Methodik}

Die Erhebung der Einschätzungen betroffener Patienten erfolgte mit fokussierten, leitfadengestützten Gruppendiskussionen. Diese (semi-)qualitative Methode stellt ein Verfahren dar, welches nicht ausschließlich hypothesenprüfend vorgeht, sondern den jeweilig Befragten ausreichend Raum gibt, eigene relevante Überlegungen, Deutungen und Themen im Hinblick auf den Stimulus - in diesem Fall die Kostensensible Leitlinie - zu fokussieren. Dabei spielt die Einzelmeinung weniger eine Rolle, als der geteilte Erfahrungsraum. Wir sind davon ausgegangen, dass die Patienten auf eine ähnliche Krankheitsgeschichte, ähnliche Behandlungen und damit auch auf ähnliche Erfahrungen zurückblicken können; aus diesem Grund schien uns ein Interview mit mehreren Patienten gleichzeitig von Vorteil.

Da die KSLL ein gewisses medizinisches Verständnis erfordern, sollten Patienten befragt werden, die an der jeweiligen Erkrankung leiden, für die die KSLL entwickelt wurden. Bei der Wahl zwischen Patienten mit einer Herzinsuffizienz (KSLL für den Einsatz eines implantierten Kardioverter-Defibrillator, ICD) und Patienten mit einer koronaren Herzerkrankung (KSLL für den Einsatz Medikamente-freisetzender Stents, DES) haben wir uns für die letztere Patientengruppe entschieden. Patienten, die für eine ICD-Implantation infrage kommen, befinden sich aufgrund der weit fortgeschrittenen Herzinsuffizienz und Begleiterkrankungen in der Regel in einem schlechten körperlichen Allgemeinzustand, sodass ihnen eine Gruppendiskussion im Rahmen der vorliegenden Studie kaum zuzumuten ist. Patienten mit einer koronaren Herzkrankheit (KHK) verfügen hingegen in der Regel über eine gute Leistungsfähigkeit, wenn sie erfolgreich mit einem Stent oder einer Bypass-Operation behandelt wurden. Zudem lassen sich Patienten mit einer KHK gut über Herzsportgruppen finden und auf die Studienteilnahme ansprechen.

Als Fokus für die Gruppendiskussionen entwickelten wir eine allgemeinverständliche Patientenversion der DES-KSLL und eine ebenfalls auf medizinische Laien abgestimmte allgemeine Einführung in das Modell der KSLL (vgl. Kap. 6.4 u. 6.5). Unter der Annahme, dass sich die Einschätzungen von Patienten aus den 16 Bundesländern nicht wesentlich unterscheiden, richteten wir unsere Anfragen an Herzsportgruppen, die sich in einem erreichbaren Umkreis der Untersucherin (SR) befanden. 
Wir kontaktierten die Landesverbände im Behinderten- und Rehabilitationssport zweier Bundesländer und baten um Unterstützung bei der Rekrutierung von Studienteilnehmern. Diese haben die Aufgabe, die Interessen der regionalen Sportgruppen zu vertreten, und damit Zugang zu allen verfügbaren Adressen. Über diesen Weg konnte lediglich ein Kontakt hergestellt werden, da die Landesverbände aus Zeit- und Kapazitätsgründen unsere Anfrage nicht prioritär bearbeiten konnten.

Zusätzlich dazu kontaktierten wir zo Herzsportgruppen in diesen zwei Bundesländern direkt per Telefon. Bei Interesse und Bereitschaft des Sportgruppenleiters wurden die Kostensensible Leitlinie zu den DES, eine Beschreibung der Studie und ein Brief an die Patienten per E-Mail und per Post versendet. Die Sportgruppenleiter haben unsere Anfrage an „ihre“ Patienten weitergetragen und Interesse an der Teilnahme festgehalten. An ihnen lag auch die Terminvereinbarung mit der Sportgruppe. Die Gruppendiskussionen sollten möglichst zu einem bekannten Termin (meist vor oder nach dem regelmäßigen Sport) in den Räumen des Vereins stattfinden, damit die Studienteilnehmer so wenig zusätzlichen Aufwand wie möglich haben. Wir bezahlten den Teilnehmern eine Aufwandsentschädigung in Höhe von 20 Euro sowie den Vereinen eine Spende von 50 Euro für die organisatorische Unterstützung.

Als Fokus für die Diskussionen wurde die DES-KSLL vorab an die Studienteilnehmer versendet. Der von uns eingesetzte Leitfaden für die fokussierten Interviews mit herzkranken Patienten (siehe folgende Aufzählung) basierte auf den Ergebnissen der vorangegangenen Untersuchungen und rahmte die Gespräche.

\section{Leitfaden für die Patienteninterviews}

- Zu Beginn möchten wir Sie bitten, uns Ihren ersten Eindruck zu schildern, den Sie beim Lesen der KSLL hatten. Erzählen Sie doch bitte, was Ihnen zuerst aufgefallen ist.

- Gibt es Aspekte, die Ihnen zu kurz kommen/die Ihnen dabei nicht so wichtig erscheinen?

- Gibt es Neues, was Sie vorher in dieser Art und Weise noch nicht gesehen haben?

- Können Sie sich vorstellen, dass diese KSLL einen Beitrag zur Lösung relevanter Probleme im Gesundheitswesen leisten könnten?

- Welche Erleichterungen/Belastungen könnte ein Einsatz der KSLL zur Folge haben?

- Was würde die Akzeptanz der KSLL Ihrer Meinung nach beeinflussen?

- Was würde die Praktikabilität der KSLL Ihrer Meinung nach beeinflussen?

- Wie schätzen Sie die Verbindlichkeit solcher KSLL ein? Nach welchen Kriterien sollten gegebenenfalls Ausnahmen möglich sein?

- Wer sollte KSLL für das deutsche Gesundheitswesen erstellen?

- Möchten Sie noch etwas ergänzen? 
Die offene Fragestellung ermöglicht eine Themenfokussierung durch die jeweiligen Interviewpartner, eigene Gewichtungen können mit dieser Erhebungsmethode deutlicher werden als mit einem strukturierten Fragebogen. Zugleich können Themen angesprochen werden, die im Leitfaden keinen Eingang gefunden haben, was zusätzliche Informationen zur Hypothesenbildung liefert. Es können durch ein solches Verfahren jedoch keine Aussagen hinsichtlich der Verteilung und der Häufigkeiten getroffen werden.

Die Diskussionen wurden im Zeitraum Mai-August 2010 von einer Mitarbeiterin (SR) durchgeführt. Insgesamt konnten wir 6 Diskussionen mit 23 Teilnehmern führen, wobei die Dauer zwischen 45 und 6o Minuten variierte. Die Gespräche wurden aufgezeichnet und vollständig transkribiert. Eingeleitet wurden die Interviews mit einer offenen Frage nach dem ersten Eindruck beim Lesen der KSLL, nach Auffälligem, nach Neuem und nach kritischen Überlegungen. Sofern die Themen des Leitfadens nicht selbstläufig angesprochen wurden, sind diese in offener Fragestellung zusätzlich nachgefragt worden. Am Ende des Interviews gab es die Möglichkeit, Wichtiges noch zu ergänzen, ein Resümee zu ziehen oder eine abschließende Bemerkung hinzuzufügen.

Die Auswertung erfolgte nach der Inhaltsanalytischen Methode (Mayring 2008), wobei die Anmerkungen, Hinweise und Darstellungen der Interviewpartner in einem Klassifikationssystem mit Ober- und Unterkategorien zusammengefasst wurden. Die Erstellung der deskriptiven Kategorien erfolgte einerseits deduktiv durch die Vorgabe der Leitfaden-Kategorien und zusätzlich induktiv aus den Gesprächen. In einer schrittweisen Auswertung wurden aus jeder Transkription Kategorien gebildet, die abschließend zusammengefasst wurden. Diese vorläufige Sammlung von Ober- und Unterkategorien wurde von drei Projektmitarbeitern (GM, DS, SR) unabhängig voneinander hinsichtlich der Cültigkeit (Validität) geprüft, Nichtübereinstimmungen wurden diskutiert; bei Bedarf wurde das Kategoriensystem nochmals geordnet und modifiziert.

\subsection{Ergebnisse}

Insgesamt gingen 364 Aussagen in die Auswertung ein, die Einschätzungen zur KSLL-DES als Instrument einer ethisch vertretbaren Rationierung für das deutsche Gesundheitswesen enthielten. Die Diskussionsmöglichkeit wurde von vielen Teilnehmern aber auch dazu genutzt, sich über ihre Erfahrungen mit der Erkrankung auszutauschen und über die jeweiligen Krankheitsverläufe und medizinischen Behandlungen zu berichten. Diese Aussagen wurden in das Kategoriensystem nicht aufgenommen, da das Ziel unserer Untersuchung in der Evaluierung der KSLL bestand. Dennoch schien es den Teilnehmern offenbar wichtig zu sein, auch über andere Erfahrungen sprechen zu können; dies wurde von der Untersucherin nicht unterbunden oder abgebro- 
chen, sondern als wichtiges Anliegen der Patienten wahrgenommen und respektiert.

Nachfolgend werden Hauptkategorien mit den jeweils dazugehörenden Unterkategorien beschrieben sowie ausführlich anhand von Beispielsätzen expliziert. Die dabei vorgenommene Ordnung der Darstellung (s. Tab. 34) stellt keine Hierarchie im Sinne von wichtig zu unwichtig dar, sondern orientiert sich eher an der Unterscheidung von formalen und inhaltlichen Gesichtspunkten; gleichwohl ergibt sich aus den Reaktionen keine strikte Trennung, sodass beide Bereiche überlappen.

\section{Tab. 34 Haupt- und Unterkategorien}

\begin{tabular}{|c|c|}
\hline Hauptkategorien & Unterkategorien \\
\hline \multicolumn{2}{|l|}{ Einschätzung der Rahmenbedingungen } \\
\hline \multirow{5}{*}{$\begin{array}{l}\text { aktuelle Versorgungssituation } \\
\text { in Deutschland }\end{array}$} & medizinische Versorgung und Behandlung \\
\hline & Zweckmäßigkeit und Wirtschaftlichkeit \\
\hline & Rationierungsbefürchtungen \\
\hline & finanzielle Eigeninteressen der Leistungserbringer \\
\hline & Ungleichbehandlung durch Trennung GKV und PKV \\
\hline \multirow{3}{*}{ Sparpotenzial/Alternativen zu KSLL } & Kostenverursacher (Industrie/Pharmafirmen) \\
\hline & Prävention, Gesundheitserziehung und Eigenverantwortung \\
\hline & Rationalisierung \\
\hline
\end{tabular}

\begin{tabular}{|c|c|}
\hline \multirow{4}{*}{ Methodik } & Erkenntnisstand und Wirksamkeit der Behandlung \\
\hline & Betroffenheit und Risiken für Patienten \\
\hline & Folgekosten \\
\hline & Generalisierung/Gesamtsituation \\
\hline \multirow{3}{*}{ Verständlichkeit } & Formulierung der KSLL \\
\hline & Informiertheit und Bildungsgrad des Patienten \\
\hline & Reaktion auf Fragestellung \\
\hline \multirow{2}{*}{ Verbindlichkeit } & Entscheidungsfreiheit des Arztes \\
\hline & Empfehlungs-Charakter \\
\hline \multirow{2}{*}{ Ersteller der Leitlinien } & medizinische Expertise \\
\hline & Gremium aus verschiedenen Experten \\
\hline $\begin{array}{l}\text { Machbarkeit/Praktikabilität/ } \\
\text { Umsetzung }\end{array}$ & private Zuzahlung \\
\hline $\begin{array}{l}\text { Funktion der Kostensensiblen } \\
\text { Leitlinien }\end{array}$ & Diskussionsanstoß \\
\hline
\end{tabular}




\begin{tabular}{ll}
\hline $\begin{array}{l}\text { Hauptkategorien } \\
\text { inhaltliche Gesichtspunkte }\end{array}$ & Unterkategorien \\
\hline \multirow{3}{*}{ Kriterien der Rationierung } & KSLL-Kriterien zu eng \\
\hline & Folgekosten und Risiken für den Patienten \\
\hline Gesamtzustand des Patienten \\
\hline Aufklärung und Transparenz & Alter \\
\hline Arzt-Patient-Beziehung & allgemein (erwünscht/nicht erwünscht) \\
\hline Belastung/Erleichterung & Vezogen auf KSLL \\
\hline Vür den Arzt & Belastung durch Verantwortungsverschiebung \\
\hline & Erleichterung aufgrund der Studienergebnisse \\
\hline Akzeptanz & Konsequenzen für Patienten \\
\hline & Information des Patienten \\
\hline Interessen der Patienten \\
\hline
\end{tabular}

\subsubsection{Einschätzung der Rahmenbedingungen}

\section{Aktuelle Versorgungssituation in Deutschland}

Die Patienten schätzen die medizinische Versorgung und Behandlung allgemein eher negativ ein. Politische Regelungen innerhalb der Gesundheitsversorgung werden als zu kurzfristig wirksam beschrieben, es fehle ihnen an Nachhaltigkeit. Niedergelassene Ärzte würden Patienten nicht mehr aufnehmen, da ihr Budget für das jeweilige Quartal erschöpft sei. Die Patienten kritisieren vor allem eine Ungleichbehandlung in Form einer Zwei-Klassenmedizin und stellen fest, dass Beziehungen zu Fachärzten eine entscheidende Bedeutung hätten, um an zeitnahe Termine zu gelangen.

„Und wenn man da nicht Beziehungen hat irgendwie, [klopft], kommt wieder dieses Vitamin B dazu, dass jemand einen kennt und sagt: na ich schieb dich da mal mit rein. So, da hat man keine Chance (...) Das ist natürlich auch schon eine Zwei-Klassen-Ceschichte“ (TN7, 168, 170).

„Da macht man zu wenig. Irgendwie ist das alles ein bisschen, leider Gottes in der großen Politikja auch, dass das ja krampfhaftes Reagieren ist aber kein längerfristiges und wirklich mit Wirkungen, die zwar nicht sofort (...) aber dann längerfristig (wirken)“( TNg, 52, 54).

Dem gegenüber beurteilen sie erlebte Doppeluntersuchungen (Hausarzt und zusätzlich Krankenhaus) oder das Verschreiben teurerer Medikamente dahingehend, dass es bei den Entscheidungen vor allem um Wirtschaftlichkeit für die 
jeweilige Klinik oder den niedergelassenen Arzt ginge und weniger um die Durchführung einer medizinisch indizierten Maßnahme.

„Aber wie gesagt, die wollen alle dran verdienen. Nach einer Untersuchung kommt wieder ein bisschen Geld rein“" (TN3, 145).

„Ja aber aus Erwerbsgründen. Weil das Krankenhaus (...) der Mediziner, der ja niedergelassen ist, der ist ja ein Unternehmer heute. Das ist ja nun mal so. (...) Der Unternehmer muss zusehen, dass er Plus macht. Also demzufolge wird er noch eine Untersuchung dranhängen, wenn's irgend geht. Das ist im System auch begründet“ (TN1, 140, 142).

Zusammenfassend resümieren die Patienten, dass das deutsche Gesundheitssystem nicht effizient sei, vor allem im Vergleich zu den Systemen anderer Länder und in Relation zu den Ausgaben und Kosten, die das Gesundheitswesen verursacht.

„Ja, es gibt ja statistisch Länder die weniger für das Gesundheitswesen pro Kopf der Bevölkerung aufwenden und die Lebenserwartung der Leute ist länger. Das zeigt, dass das deutsche Gesundheitswesen nicht spitze ist“ ( $\mathrm{TN} 1,205)$.

\section{Sparpotenzial/Alternativen zu KSLL}

Diese Kategorie weist Überschneidungen mit der Kategorie Akzeptanz auf, da es sich hierbei um die Einschätzung des Nutzens der exemplarischen KSLL hinsichtlich einer Kostenersparnis im Gesundheitswesen handelt. Dennoch haben wir bestimmte Aussagen dieser Kategorie zugeordnet, da die Patienten hier Alternativen zu dem diskutierten Instrument einer expliziten Rationierung aus ihrer Sicht benennen.

So scheint der allgemeine Tenor der Aussagen zu sein, dass eine Rationierung an der „letzten“ Stelle, also am konkreten Patienten nicht zu akzeptieren sei. Vielmehr sollte man bei den Kostenverursachern ansetzen, bei denen sich aus Sicht der Patienten ein wesentlich größeres Einsparpotenzial realisieren ließe. Beispielsweise greift ein Patient die Differenz zwischen den beiden Stent-Behandlungen auf und vergleicht sie mit den Gehältern der Krankenkassen-Geschäftsführern. Demzufolge sei diese Differenz marginal und führt eben nicht zur Verringerung von Kosten, sondern setzt am falschen Ende an.

So finden sich vor allem Einschätzungen alternativer Kostenverringerung in Form von Begrenzung von Gehältern, Festlegung von Maximalpreisen für Medikamente und Regulierungen der Pharmafirmen.

„Bisher konnteja die Industrie ihre Preise weitgehend selber bestimmen. Da ist ja nun ein großer Rummel, dass das geändert werden soll aber solange die Abgeordneten unterstützt werden von der Industrie, damit sie in den Bundestag kommen, solange ist mein Claube an die Wahrhaftigkeit des Willens kaum vorhanden, ja. Und wieso geht man da nicht an die Industrie heran und nicht beim Patienten, derja schließlich 
und letzten Endes erstens nicht der Fachmann dafür ist und zweitens ja eigentlich davon profitieren sollte mit seiner Gesundheit“ (TN11, 8).

„Und wenn wir beim Sparen sind, eigentlich sollte man dort anfangen mit dem Sparen, wo die Kosten entstehen. Die entstehen bestimmt nicht beim Patienten“(TN11, 4).

„Die Kosten entstehen doch politisch.Welche Beschlüsse gibt's, welche Gesetze gibt's, wer traut sich, welche Preise festzulegen, ohne dass es irgendeiner kontrolliert. Was weißich“ (TN1, 71).

Eine weitere Möglichkeit der Einsparung von Kosten im Gesundheitswesen sehen die Patienten in einer stärkeren Fokussierung auf Prävention und Gesundheitserziehung, also einer langfristigen und nachhaltigen Umerziehung, die dann Folgekosten, in Form von bspw. Herz-Kreislauf-Erkrankungen, sparen helfen könnte. Auch die Stärkung der Eigenverantwortlichkeit eines (Nochnicht-)Patienten könnte einen Beitrag zur Minimierung von Folgekosten leisten.

„Man muss aber den Patienten auf jeden Fall irgendwie mit als, ich weiß nicht, ob jeder selbst natürlich bereit ist, seine Mitwirkungen zu ermöglichen und damit vielleicht der Sache sogar mehr Einsparungen zu bringen, als irgendwelche Vorgaben“ (TN9, 54).

„Und ich denke mal, vielleicht auch noch ein ganz wichtiger Moment ist hier bei dieser ganzen Geschichte, dass vielleicht auch gezielte, also Präventionen auf jeden Fall ... dass es gar nicht erst zu so einem Vorfall kommt (...) und gezielte Aufklärung (stattfindet)“ (TN7, 60).

\subsubsection{Formale Gesichtspunkte}

\section{Verständlichkeit}

Die Verständlichkeit der von uns erarbeiteten Patientenversion der KSLL wurde von den Patienten unterschiedlich eingeschätzt. In einer Gruppe waren sich alle darüber einig, diesen Text nicht verstanden zu haben, vor allem, was die medizinischen Begrifflichkeiten und die Rechnung der Kosteneffizienz (unter Einbezug der QALY-Werte) betraf. Auf Nachfrage wussten einige der Patienten auch nicht, welche Art von Stent sie eingesetzt bekamen.

„Ich denke: waswollen die denn eigentlich? (...) hier geht's um Kosten, Kostensenkung, nö, ich hab's nicht verstanden“ (TN3, 86).

In den anderen Gruppen hingegen wurde den KSLL eine allgemeine Verständlichkeit zugesprochen. Dies sei durch ein Interesse der Patienten an der Erkrankung und deren Behandlung sowie ein Informationsbedürfnis bedingt, was sich auch ausdrückt im Beschaffen weiterer Informatiotnen neben der ärztlichen Beratung und Aufklärung. 
Dass ein Verstehen nicht unabhängig vom Bildungsgrad der Betroffenen ist, kann hierbei angenommen werden.

„Es kann natürlich sein, es hängt auch ein bisschen so vom Bildungsgrad ab, dass vielleicht es doch einige Patienten gibt, die schon mal ein bisschen Schwierigkeiten mit einigen Fachbegriffen haben“ (TN10, 68).

„Na man informiert sich allgemein auch durch Lesen von Fachzeitschriften oder allgemein Presse oder sieht man im Fernsehen sehr viele detaillierte Berichte, die man sich auch mit Interesse anguckt als sagen wir mal teils Patient, Betroffener, sodass das eigentlich verständlich war und man weiß, worum es geht“"(TN10, 58).

An der Formulierung der KSLL fanden die Patienten einige Kritikpunkte, die zum einen die Verwendung von Abkürzungen betreffen, die eine Lesbarkeit erschweren. Aber auch die Formulierung, dass der vorenthaltene Nutzen den Patienten am „wenigsten weh tun“ darf, halten die Patienten für psychologisch unglücklich. Mit diesem Ausdruck würde den Patienten eher vermittelt, dass ihnen Schaden vorenthalten werden sollte, statt geringfügiger Zusatznutzen.

„Daslese ich natürlich nicht, dass es wenig nutzt, sondern dasses am wenigsten schadet. So. Dass man den Schaden minimiert. So lese ich das, ja“ (TN11, 300).

\section{Verbindlichkeit}

Die Frage nach der Verbindlichkeit solcher Kostensensiblen Leitlinien wird von den Patienten in Richtung Empfehlung beantwortet. Kein Patient sah es als notwendig an, Ärzten eine Richtlinie vorzuschreiben, nach der sie sich ohne Ausnahmen zu verhalten hätten. Wenn man sich die allgemeine Einschätzung der Patienten bezüglich des Gesundheitssystems anschaut, würde hier eher eine Zustimmung zu einer hohen Verbindlichkeit erwartet werden, um Einzelfallentscheidungen eines Krankenhauses oder Arztes ausschließen zu können und einer Ungleichbehandlung entgegenzuwirken.

„Es darf nicht verpflichtend sein für den Arzt, er muss immer die Wahl haben. Es können nur Richtlinien sein. Das muss nicht festgezurrt sein, dass er sich danach richten muss. Denn das würde ja sage ich mal das könnte ihn einschränken, den Mediziner“ (TN1, 253, 255).

„Die darfkein Dogma sein“(TN12, 107).

Jedoch auf der spezifischen Arzt-Patient-Ebene berichten die Patienten weitaus positivere Erfahrungen (siehe Kategorie Arzt-Patient-Beziehung) und stellen die Entscheidungsautonomie des Arztes in den Vordergrund. Diesem dürften keine Einschränkungen gemacht werden.

„Das ist nur ein grobes Richtmaßund den muss er noch weiter detailliert dann untersuchen“"(TN12, 155). 
„Er muss in seiner Entscheidung einen Spielraum haben. (...) Denn das ist nicht alles“ (TN11, 154).

Der Arzt müsse aus verschiedenen Faktoren und Bedingungen der je spezifischen Situation des Patienten und aufgrund seiner Erfahrungen über die indizierte Methode/Behandlung entscheiden dürfen. Ausnahmen sollten immer aufgrund der Gesamtsituation des Patienten möglich sein.

„Das kann eine grobe Richtlinie sein aber man muss dann noch tiefer gehen, ja. wie ist die Konstitution des Einzelnen ..." (TN12, 100).

„Ja wir sind nicht von der Stange, wir Menschen sind unterschiedlich. Ja“ (TN11, 102).

\section{Ersteller der Leitlinien}

Die medizinische Expertise sollte für die Erstellung der KSLL maßgeblich sein. Eine nachgeordnete, aber wichtige Funktion räumen die Patienten ökonomischen und ethischen Überlegungen ein. Eine Beteiligung von Patientenvertretern sehen die befragten Patienten als einen wichtigen Bestandteil bei der Ausarbeitung und Formulierung solcher Leitlinien.

„Also es sollten grundsätzlich nicht nur Ökonomen am Werk sein. (...) Also es müssten in jedem Fall zuerst die Ärzte sein, die (...) Ärzte, die sicher dann aus der Erfahrung wissen, wo man die einfacheren Mittel gut einsetzen kann, wirksam einsetzen kann und wo notwendig sind die, ich sage mal hier die ... teureren" (TN10, 207, 210).

Zusammenfassend lässt sich also sagen, dass die Patienten ein Gremium mit vorrangig ärztlicher Expertise wünschen, welches sich mit der Erstellung von Leitlinien für das Gesundheitssystem beschäftigt.

\section{Umsetzung der Leitlinien}

Sofern die KSLL maßgeblich für die Kostenübernahme der Gesetzlichen Krankenversicherung würden, sollte es die Möglichkeit geben, die durch die Leitlinie rationierte Behandlung mittels einer privaten Zuzahlung zu erhalten. Es sei zwar ein schlechterer Weg als eine prinzipielle Gleichbehandlung aller Patienten, aber so hätte man als Patient die Freiheit, sich finanziell aus dieser Ungleichbehandlung rauszukaufen.

Hier wird vor allem der Wert der Gesundheit betont, den jeder Einzelne ihr beimisst und in dessen Entscheidung es dann auch läge, wofür er sein Geld ausgeben möchte. Diese Entscheidung dürfte nicht reglementiert werden, sondern sollte eine Wahloption für den Patienten beinhalten.

„Also ich würde dann, wenn es keine andere Möglichkeit gibt, würde ich sogar als Patient noch einen Schritt weiter gehen und sagen: jawohl, wenn's keine andere Möglichkeit gibt, ich möchte aber das Optimale und ich muss vielleicht dann 200 Euro zu 
bezahlen, wäre ich bereit für mich, für meine Gesundheit 200 Euro noch dazu zu bezahlen“ (TN4, 229).

„Auch wenn man nun mal, das ist die Politik, nun unbedingt hier sparen will, dann sollte man aber auch den Patienten die Möglichkeit geben, die jetzt aus dem Raster herausfallen, dass sie den 800 Euro Stent nicht bekommen, die 400 Euro zuzahlen, um ihn zu bekommen“ (TN12, 40).

\section{Funktion der KSLL}

Hinsichtlich der Funktion der KSLL als ein Instrument expliziter Rationierung sahen es die Patienten als kritisch an, dass sie möglicherweise mit der wissenschaftlichen Entwicklung nicht würden Schritt halten können. Sie sehen das Potenzial der KSLL maximal darin, die Diskussion über die Kosten im deutschen Gesundheitswesen verursachen zu können.

„Aber ich sage mal, diese Kostenfrage, wird die nicht ständig konterkariert durch die ganze Geschichte der Wissenschaft, wo jeden Tag irgendwas Neues kommt. Bei den vielen Leuten, die in der Wissenschaft arbeiten, sind Sie mit Ihren Kosten immer hinten dran. Das ist wie die Kripo hinter dem Verbrecher. Die schaffen das auch nie, die Brüder zu fassen, weil die sich auch immer was Neues ausdenken. Und hier sehe ich das genauso“ (TN1, 19).

\subsubsection{Inhaltliche Gesichtspunkte}

\section{Kriterien der Rationierung}

Die in der KSLL aufgeführten Kriterien für die Indikationseinschränkungen betrachten die Patienten als unverständlich und nicht ausreichend.

„Für manche reicht der normale Stent aus, aber der andere ist wieder (...) aber dasjetzt nach technischen Parametern und nach Millimetern, das ist natürlich ein bisschen kritisch, das ist zu einfach, zu simpel, ja“ (TN12, 92).

„Ich verstehe nur nicht die Auswahl, wieso bei einem bestimmten Durchmesser das eine gilt und das andere nicht mehr und umgekehrt. Länge oder Durchmesser, das wollen wir mal gleich setzen. Wonach diese Werte bestimmt worden sind, auf welcher Grundlage? Dass das eine eingesetzt wird und das andere nicht“" (TN11, 4).

Sie bemängeln die Ausblendung von Folgekosten, die mit der rationierten Behandlung auf das Gesundheitssystem zukommen könnten. Ebenso sehen sie auch die Risiken für die Patienten als zu wenig beachtet, was ihnen ein wichtiger Faktor zur Entscheidung für oder gegen eine Behandlung sei.

„Wenn es bewiesen ist, dass das eine besser ist und sagen wir mal die Summe der Kosten sagen wir mal vergleichbar machen, dann sollte man aufjeden Fall das auch sehen, wie der Einzelne damit betroffen ist, wenn er mehrfach diesen Stent da gesetzt bekommt und den Eingriff machen muss, die Untersuchung und mit den Problemen, die 
dabei entstehen können. Und die sind oft sagen wir mal sicher auch kostenmäßig erheblich“(TNg, 56).

Weiterhin wünschen sich die Patienten bei Rationierungsentscheidungen die Berücksichtigung des Gesamtzustandes des Patienten und nicht nur Kriterien, die sich auf Merkmale der Stenose des Gefäßes beziehen.

„Darum sagte ich ja schon, wir sind nicht von der Stange. Die Menschen sind unterschiedlich. Ja, das kann man nicht generalisieren“"(TN11, 159).

Auch die Frage, ob das Alter ein Kriterium der Rationierung darstellen sollte, wird von den Patienten diskutiert und eingeschätzt. Ein Patient führt die Überlegung an, dass es vor allem im Zuge der hochtechnisierten Medizin und der Möglichkeiten, die sich daraus ergeben, ethisch vertretbar sei, nicht mehr Medizin um jeden Preis zu praktizieren, und somit das Alter, aber wahrscheinlich vornehmlich den Gesamtzustand des Patienten als Kriterium des Vorenthaltens von Maßnahmen heranzuziehen.

„Und das ist jetzt, sage ich mal, ein ganz böser Satz, den ich jetzt ausspreche so. Aber ich hab es erlebt, als ich auf der Intensivstation gelegen habe, da war auch einer, der war 87 oder 88 und der musste gefüttert, gewaschen werden und hing an der Nierenwaschanlage, wie heißt das ... (Dialyse) (...) das ist jetzt hart, wasich sage, aber wenn einer mit der Gesundheit so fertig ist, der nur noch liegen kann und gedreht werden muss und mit dem Hubbett und der muss dann eingerieben werden, dass die Haut nicht wund gelegen wird und der muss dann noch beatmet werden, dass er keine Lungenentzündung kriegt und alles und so, dass man dann vielleicht doch lieber sagt ... ich weiß nicht, es gibt eine humane Lösung, wir lassen ... ich sag es jetzt mal ganz offen: wir lassen so einen Menschen einschlafen“"(TN7, 136, 138).

Alle anderen Patienten sahen gerade dieses Kriterium als sehr kritisch und befürworteten es nicht.

„Nun sollte man um Gottes Willen nicht vom Alter ausgehen. Die Patienten sind von bis... das ist keine alleinige Altersfrage,... Also das ist keine Altersfrage. Ich möchte warnen, das zum Beispiel an die Altersfrage zu koppeln“ (TN11, 130).

\section{Aufklärung und Transparenz}

Hinsichtlich der Frage der Aufklärung und Information der Patienten über die Behandlung nach bestimmten Leitlinien, geben die Patienten an, dass sie das dazu erforderliche Wissen nicht hätten. Vor allem, wenn es um Notfälle ging (viele der Patienten erhielten den Stent infolge eines akuten Ereignisses), könne der Arzt den Patienten nicht noch aufklären.

„Vor allem ist das Ding gegessen, wenn der das Ding einpflanzt, dann habe ich das drin. Ob da hinterher noch was lese oder nicht lese. (...) Denn der Arzt entscheidet ja, was ich rein kriege“ (TN4, 154). 
„Ich bin zum Arzt gegangen, ich sage: mir ist ein bisschen komisch. Und dann EKG und sofort ins Krankenhaus und zwei Tage später war ich operiert, ja, da hat man sowieso keine Wahl mehr ja“" (TN13, 283).

Die Patienten zogen auch in Betracht, dass sich die Ärzte davor scheuten, die Patienten über das Vorenthalten einer, wenn auch geringfügig, nützlichen Maßnahme zu informieren.

„Na ja, der Arzt sagt: ja, ich will hier keine schlafenden Hunde wecken. Und der hat auch Angst, diese ganze Geschichte mit diesen Sachen das ist ja auch psychologisch muss man da also auch gut sein und das muss man auch erst mal verarbeiten. Und die haben da Angst, wenn man... also wenn man zu viel weiß vielleicht oder so ..." (TN7, 114).

„Wenn man beim Arzt ist und der guckt den Patienten nicht an und eigentlich sagter: ja, ich könnte ihnen das verschreiben, das ist aber so teuer, das geht nicht und wir müssen ausweichen aufein billigeres. Ist psychologisch nicht gerade klug“ (TN10, 203).

Aufklärung über die Erkrankung und Behandlung wird von Patienten aber auch gewünscht und erwartet. Sie betonen hierbei die Möglichkeit einer Mitarbeit des informierten Patienten.

„Ich möchte schon wissen, warum ich operiert werden muss und warum dies und warum jenes gemacht werden muss, immer. Ich frag da grundsätzlich nach, wenn ich nicht genug Informationen kriege, immer. Das ist mein Körper ist das, ja, ich möchte schon wissen, was da mit mir passiert" (TN3, 291).

\section{Arzt-Patient-Beziehung}

Wenn die Patienten von individuellen Erfahrungen mit ihren behandelnden Ärzten berichten, ist die Einschätzung weitaus positiver als bei der allgemeinen Einschätzung der Versorgungssituation in Deutschland. Sie fühlen sich in ihrer Erkrankung optimal behandelt, haben Vertrauen zu den Ärzten und schätzen deren Arbeit. Solche Leitlinien würden vor allem das Vertrauen zwischen Arzt und Patient untergraben.

„Denn der ist abhängig von dem Ratschlag oder von der Tätigkeit des Arztes. Und der Patient hat da keinen Einfluss drauf, der muss sich daraufverlassen, dass der Arzt das beste für ihn tut. Und man sollte sich hüten, durch solche Dinge das Vertrauen zwischen Patienten und Arzt zu belasten" (TN11, 14).

„Letztendlich ist das alles für mich auch eine große Vertrauenssache, ja. Das Vertrauen zu dem Arzt oder zu den Ärzten. Wenn sie sagen in München oder in Hamburg oder wo ich gerade bin oder so, ich muss dieses Vertrauen, was ich bis dahin bei meinem speziellen Arzt mussich auch zu diesem Arzt dann mitnehmen. Und da gehe ich auch so ran. Aber wenn mir dann so entgegen kommt, hier, das ist so teuer oder was auch immer. Vielleicht kommen noch solche Äußerungen mir persönlich richtig gegenüber, 
dann ist doch das futsch, dann ist das für die Katze. Und es kommt letztlich wieder aufden Patienten zurück" (TN4, 121).

\section{Belastung/Erleichterung für den Arzt}

Die einzige Erleichterung, die die Patienten durch die Behandlung nach einer KSLL für den Arzt sehen, sei die Beweislage durch die Studien. Diese könnten den Arzt stützen in seiner Entscheidung für oder gegen eine Behandlung.

„Na ja, die andere Seite ist aber die. Es ist auch schön, wenn der Arzt eine Richtlinie hat, wo er weiß, dass dort schon mehrjährige Studien sind und ganz umfangreiche Untersuchungen waren und der weiß: aha, also. Es stand ja mal drin: an der Stelle erweist sich das als das günstigste oder so, ja“"(TN13, 323).

Weitaus eher sehen die Patienten durch eine Verantwortungsverschiebung des Arztes in seiner Entscheidung eine Belastung. Der Arzt könnte durch eine Leitlinie unter Druck geraten, sich danach verhalten zu müssen, wenn ihm keine Entscheidungsautonomie zugestanden würde.

„Und wenn der Druck der Kosteneinsparung vielleicht so großwird, dass dem Arzt die Hände gebunden sind ...(...) wird fast gezwungen, so zu handeln“ (TN10, 194).

„Ja aber das ergibt auch dann wieder eine Möglichkeit für den Arzt, sich dahinter zu verstecken“(TN11, 26).

\section{Akzeptanz}

Wie nun schätzen die Patienten die Akzeptanz einer expliziten Leistungssteuerung mittels KSLL ein? Hier zeigen sich verschiedene Gesichtspunkte, die für die Patienten relevant sind. Zum einen spielt es eine entscheidende Rolle, welche Konsequenzen die Behandlung nach dieser Leitlinie für sie habe.

„Na ja, wenn er ... ich meine, wenn die Möglichkeit, also die technische Möglichkeit besteht, dass der Patient ich sage mal dann keine gesundheitlichen Folgen hat anschließend, das steht ja hier auch drin (...) wenn der jetzt sage ich mal ein halbes Jahr oder so das Teil drin hat und dann liegt er wieder auf dem Tisch und dann muss er vielleicht doch operiert werden oder muss einen Bypass bekommen und ich weiß, dass so eine Herzoperation ungefähr 20000 Euro kostet" (TN7, 63).

Eine Erhöhung der Akzeptanz sei beispielsweise durch die Aufklärung des Patienten zu erreichen. Jedoch spielen die Interessen des einzelnen Patienten wohl eine größere Rolle. Wenn es um die eigene Betroffenheit geht, sind sich die Patienten einig in ihrer Einschätzung, dass sie eine geringer nützliche Behandlung für sich nicht akzeptieren würden.

„Ich als Patient, wenn ich das so lese ... was interessieren mich die Kosten? Ich gehe ja ... mir soll geholfen werden, Punkt, aus. Lass das doch kosten, was will. Das ist jetzt mein Gedanke dabei“(TNi4, 91). 
„Ich wollte die bestmögliche Behandlung, die bestmögliche möchte ich haben/Nein ich möchte das Supergute, das Beste möchte ich haben. Ich möchte nicht ein Mittelmaß, ich möchte das Beste" (TNi3, 177/215).

\subsection{Diskussion}

Die Diskussionen mit Patienten mit einer koronaren Herzkrankheit machen deutlich, dass die Frage der Leistungssteuerung mittels KSLL nicht unabhängig von der Gesamtsituation der Gesundheitsversorgung in der GKV zu sehen ist. Insbesondere verweisen die Interviewpartner auf bereits bestehende Ungleichbehandlungen im aktuellen System, v.a. hervorgerufen durch die Trennung in das System der Privaten Krankenversicherung (PKV) und Gesetzlichen Krankenversicherung (GKV). Auch persönliche Beziehungen spielten eine große Rolle beim Zugang zu den besten Behandlungsmöglichkeiten, sodass bereits heute eine „Zwei-Klassen-Medizin“ vorherrsche. Zudem berichten die Diskussionsteilnehmer über ökonomisch bedingte Überversorgung, sowohl auf der Ebene des einzelnen Arztes als auch auf der Ebene von Krankenhäusern. Auch durch Doppeluntersuchungen im ambulanten und stationären Bereich entstünden unnötige Kosten. Die Patienten bestätigen damit einen Befund, der auch in den Befragungen von Ärzten im deutschen Gesundheitswesen deutlich wurde (vgl. z.B. Strech et al. 2008): Derzeit laufen die Zuteilungsentscheidungen intransparent, nach medizinisch wenig rationalen Gesichtspunkten ab und führen zu einer problematischen Ungleichbehandlung von Patienten. Dieser Befund war für uns ein wesentliches Argument, die KSLL als ein medizinisch rationales, ökonomisch sinnvolles und ethisch gut begründetes (unvermeidbare Leistungseinschränkungen sollten dort stattfinden, wo der Nutzengewinn für die Patienten gering ist) Modell einer expliziten Leistungssteuerung zu entwickeln.

Wenn man nun aber glaubte, die Patienten würden die ihnen im Rahmen dieser Studie präsentierten KSLL als einen vorzugswürdigen, alternativen Weg der Leistungssteuerung begrüßen, so wird man durch die Diskussionen eines Besseren belehrt. Es sei unvertretbar, mittels KSLL am einzelnen Patienten zu sparen, solange es noch alternative Möglichkeiten der Einsparung im System gäbe. Insbesondere weisen sie darauf hin, dass bei den Akteuren im System eingespart werden solle, die die Kosten verursachen: die Leistungserbringer. Die Patienten seien aufgrund des fehlenden Fachwissens kaum in der Lage zu beurteilen, welche Maßnahmen einen relevanten Zusatznutzen bieten und welche nicht. Durchaus nachvollziehbar weisen sie darauf hin, dass die Inanspruchnahme medizinischer Leistungen vor allem durch ärztliche Entscheidungen verursacht würde. Die Möglichkeiten der Einflussnahme von Patientenseite bei einzelnen Therapieentscheidungen schätzen sie eher skeptisch ein. Sie seien einem in weiten Bereichen wirtschaftlich betriebenen Medizinsystem ausgeliefert. Dies bestätigt die These, dass ein vernünftiger, kosten- 
bewusster Ressourceneinsatz es erfordert, dass Ärzte auch im Einzelfall Verantwortung für die finanziellen Implikationen ihrer Entscheidungen übernehmen - vielleicht mehr, als dies bisher der Fall ist (Marckmann $u$. in der Schmitten 2011). Die Diskussion, wie dies in einer ethisch vertretbaren Art und Weise geschehen kann und wie insbesondere mit dem möglicherweise resultierenden Konflikt zwischen den Interessen des einzelnen Patienten und den gerechtigkeitsethischen Verpflichtungen gegenüber anderen Patienten umzugehen ist, steht innerhalb der Ärzteschaft noch am Anfang.

Der Grundlinie dieser Argumentation folgend, machen die Patienten auch konkrete Vorschläge, wo im System an anderer Stelle Ressourcen eingespart werden könnten, bevor bei ihnen, dem schwächsten Glied in der Kette, Einschränkungen bei medizinischen Maßnahmen vorgenommen würden (,am Patienten sollte man zuletzt sparen“): Senkung der Gehälter der Krankenkassen-Mitarbeiter und Chefärzte, Vermeidung von Doppeluntersuchungen und ökonomisch motivierter Übertherapie sowie stärkere Preiskontrollen bei Medikamenten. Im weitesten Sinne könnte man diese Maßnahmen unter Rationalisierungsmaßnahmen fassen: Mit weniger (finanziellen) Ressourcen wird das gleiche Ergebnis bei den Patienten erzielt. Die hier befragten Patienten vertreten damit eine ganz ähnliche Position, wie die von uns befragten Experten und Interessenvertreter aus dem deutschen Gesundheitswesen (s. Kap. 9): Das Vorenthalten nützlicher Leistungen aus Kostengründen beim einzelnen Patienten („Rationierung“) ist eigentlich erst dann vertretbar bzw. akzeptabel, wenn die noch verfügbaren Wirtschaftlichkeitsreserven im System ausgeschöpft wurden. Einerseits muss man auch hier wieder entgegnen, dass es nie ein vollkommen effizientes Gesundheitssystem geben wird und man folglich mit diesem Argument dauerhaft alle Bemühungen unterlaufen könnte, die aktuell vorherrschende implizite Rationierung in eine ethisch zu bevorzugende explizite Rationierung zu überführen. Anderseits unterstreicht dieser Befund, wie dringend notwendig es auch aus Patientensicht ist, sich um die verbreitete Überversorgung im Gesundheitssystem zu kümmern und hohe Verdienstmöglichkeiten und Profite im System der GKV auf den Prüfstand zu stellen. Entscheidend ist nicht das Erreichen einer perfekten Effizienz, sondern das tatkräftige Bemühen um eine Steigerung der Effizienz - nur unter dieser Voraussetzung lassen sich Ansätze einer expliziten Rationierung legitimieren (vgl. die „benchmarks of efficiency“ von Strech u. Danis 2014). Was von den Diskussionsteilnehmern nicht gesehen wurde (dies war auch in der Einführung nicht ausdrücklich erwähnt): Die KSLL beruhen ja im ersten Schritt auf einer evidenzbasierten Nutzenbewertung und bieten damit das Potenzial, medizinische Maßnahmen ohne Zusatznutzen bzw. ohne ausreichend verlässliche Evidenzgrundlage bereits auf dieser Stufe auszuschließen. Insofern schlägt man mit den KSLL gewissermaßen zwei Fliegen mit einer Klappe: Zum einen werden Rationalisierungspotenziale genutzt und zum anderen werden die Voraussetzungen für eine ethisch vertretbare Rationierung geschaffen (s. Kap. 2). 
Zwar weisen die Diskussionsteilnehmer klar auf die Entscheidungsverantwortung der Leistungserbringer bei der Entscheidung über konkrete Maßnahmen hin, sehen aber durchaus auch die Versicherten und Patienten in der (Mit-) Verantwortung - allerdings auf einer anderen Ebene: Durch Prävention und „Gesundheitserziehung“ könnten die Versicherten mit dazu beitragen, dass spätere Folgekosten vermieden oder zumindest etwas reduziert werden.

Obgleich einige Gesprächsteilnehmer sich nicht richtig auf die KSLL einlassen wollten (schwer verständlich, Aufgabe der Ärzte), zeigten andere ein erstaunliches Verständnis für die methodischen Herausforderungen der KSLL. So wurde z.B. infrage gestellt, ob die KSLL überhaupt mit dem rasanten Wissensfortschritt würden mithalten können und ob denn wirklich die Kosten umfassend abgeschätzt seien, einschließlich der erforderlichen Begleitmedikationen und möglicher Folgekosten. Sie äußerten sich auch skeptisch, ob es möglich sei, die Komplexität und Individualität der Einzelfälle in einer solchen Leitlinie abzudecken. Die KSLL dürften deshalb nur einen Empfehlungscharakter haben und sollten den Ärzten im Einzelfall einen entsprechenden Entscheidungsspielraum offen lassen, der es ihnen ermöglicht, eine Entscheidung im besten Interesse des Patienten auf der Grundlage einer umfassenden Situationsbeurteilung zu treffen. Die Patienten äußerten sich skeptisch, ob eine Therapieentscheidung tatsächlich auf einzelne Parameter wie den Gefäßdurchmesser oder die Länge der Engstelle reduziert werden könne. Hier vertrauen sie deutlich mehr der Urteilskraft des Arztes. Das klare Bekenntnis zu legitimen Ausnahmen drückt sich auch darin aus, dass die Diskussionsteilnehmer auf jeden Fall die Möglichkeit haben möchten, sich die ausgeschlossenen Maßnahmen privat dazu kaufen zu können. Das sei zwar nicht die ideale Lösung (das wäre der gleiche, uneingeschränkte Zugang für alle), aber doch besser als ein restriktives System, das einen privaten Zukauf ausschließt.

Diese Bereitschaft zur finanziellen Eigenbeteiligung unterstreicht, wie wichtig den Teilnehmern der Zugang zur medizinischen Versorgung ist. Immer wieder wird betont, dass sie die bestmögliche Therapie haben möchten, unabhängig von den entstehenden Kosten. Dass dies schon unter den gegenwärtigen Bedingungen schwierig und zukünftig unmöglich sein dürfte, scheint den Patienten nicht bewusst zu sein - zumindest wird es von ihnen nicht erwähnt. Mit verantwortlich für diese Einstellung könnte auch die Gesundheitspolitik sein, die nach wie vor den Patienten verspricht, dass jeder alles bekommt, was er medizinisch benötigt. Hier wäre eine öffentlicher Diskurs über die Grenzen der Finanzierbarkeit des solidarisch finanzierten Gesundheitswesens hilfreich, wie er in den Ländern mit erfolgreichen Initiativen zur Priorisierung (v.a. Schweden und Norwegen) stattgefunden hat (zur Übersicht vgl. Marckmann 2009).

Diese Erwartungshaltung an die Gesundheitsversorgung würde - so die Einschätzung der Diskussionsteilnehmer - auch die Akzeptanz von KSLL im Einzelfall bestimmen: Welche konkreten Konsequenzen resultieren für den jewei- 
ligen Patienten durch die Anwendung der KSLL? Verschiedene Teilnehmer betonen, dass KSLL nicht akzeptabel seien, die ihnen einen Nutzen vorenthalten. Interessanterweise werden die KSLL aus diesem Grunde auch als eine Belastung für das Vertrauensverhältnis der Patienten zu ihrem Arzt gesehen. Bei aller Kritik an ärztlichen Entscheidungen im System, bringen die Patienten ihren jeweils „eigenen“ ̈̈rzten nach wie vor eine hohe Wertschätzung entgegen. Sie würden es gerade deshalb als einen Vertrauensverlust empfinden, wenn der Arzt ihnen nicht mehr die beste Therapie anbieten würde. Der Patient sei aufgrund der Wissens- und Informationsasymmetrie nicht in der Lage, die medizinische Entscheidung unabhängig selbst zu beurteilen. Er müsse in dieser Situation dem Arzt vertrauen können, dass er die in der jeweiligen Situation bestmögliche Therapie erhält. Unsere Hypothese, dass die Arzt-Patient-Beziehung durch die KSLL entlastet werden könne, wird durch diese Einschätzungen nicht bestätigt. Wie die Patienten selbst berichten, hängt die Auswirkung stark von der Information über die Entscheidung auf der Grundlage einer KSLL ab. Auf der einen Seite verlangen die Patienten eine entsprechende Aufklärung von ärztlicher Seite, auf der anderen Seite seien die Informationen für den Patienten oft schwer zu verstehen. Insbesondere bei akutem Behandlungsbedarf, wie er gerade bei der koronaren Herzerkrankung auftreten kann, sehen sie wenig Möglichkeiten für eine ausführliche Information über die Entscheidung, ob ein Medikamente beschichteter Stent oder ein normaler Metallstent eingesetzt werden soll. Zudem waren sie skeptisch, ob die Ärzte tatsächlich darüber aufklären würden, wenn sie einem Patienten eine teure Maßnahmen mit geringem Zusatznutzen gemäß einer KSLL vorenthalten würden, da dies vom Patienten vermutlich nicht leicht zu verdauen wäre. Eine Entlastung könnten die KSLL aber für die Ärzte bedeuten, da sie eine Entscheidungshilfe auf der Crundlage der verfügbaren Evidenz aus klinischen Studien erhalten. Allerdings mahnen die Diskussionsteilnehmer auch hier zur Vorsicht: Es dürfe nicht zu einer Verlagerung der Verantwortung kommen, wenn sich die Ärzte hinter den KSLL „verstecken“. Einmal mehr unterstreicht dies das große Vertrauen der Patienten in das unabhängige individuelle ärztliche Urteil.

Insgesamt weisen die Beiträge der Diskussionsteilnehmer darauf hin, dass die KSLL derzeit eher (noch) keine große Akzeptanz bei den Patienten finden würden. Zunächst sollte überzeugend dargelegt werden, dass im System an anderer Stelle ebenfalls Maßnahmen zur Kostendämpfung unternommen werden, insbesondere bei der ökonomisch motivierten Überversorgung. Die Initiative Choosing wisely, die aktuell auch in Deutschland zunehmend Fuß fasst, könnte hier in wichtiger Schritt in die richtige Richtung sein (Strech et al. 2014). Dann bedarf es noch weiterer Aufklärung über die genaue Funktion der KSLL in der Arzt-Patient-Beziehung, vor allem auch mit Blick auf die aktuell vorherrschende implizite Rationierung, die vielleicht im Einzelfall weniger belastend wirkt, da die Einschränkungen nicht transparent gemacht werden und in den meisten Fällen auch nicht dem Patienten kommuniziert werden. 


\subsubsection{Limitationen}

Diese Untersuchung liefert keine repräsentativen Ergebnisse im Sinne einer Verteilung oder Häufigkeit von Einschätzungen der Patienten zu den KSLL. Vielmehr versteht sie sich als Darstellung eines Spektrums möglicher Aspekte, die in die Beurteilung der KSLL aus Sicht der Betroffenen einfließen können. Dabei handelt es sich um Patienten mit einer bestimmten Erkrankung (koronare Herzkrankheit), die sich zu einer bestimmten KSLL (Medikamentefreisetzende Stents, DES) geäußert haben. Es ist nicht auszuschließen, dass andere Patientengruppen mit anderen KSLL andere oder weitere Einschätzungen äußern würden.

\section{Autorenerklärung und Danksagung}

Diese Untersuchung wurde im Rahmen des Forschungsverbunds „Allokation“ mit Mitteln des Bundesministeriums für Bildung und Forschung (BMBF) gefördert (Fkz 01GP0608). Die Verantwortung für den Inhalt dieser Veröffentlichung liegt bei den Autoren. Wir danken den Interviewpartnern für ihre Bereitschaft, uns ihre Zeit und ihr Wissen für diese Studie zur Verfügung zu stellen. Für die Transkription der Interviews bedanken wir uns bei Hendrik Bittorf.

\section{Literatur}

Marckmann G (2009) Priorisierung im Gesundheitswesen: Was können wir aus den internationalen Erfahrungen lernen? Z Evid Fortbild Qual Gesundhwes 103, 85-91

Marckmann G, in der Schmitten I (2011) Wie können Ärzte ethisch vertretbar Kostenerwägungen in ihren Behandlungsentscheidungen berücksichtigen? Ein Stufenmodell. Ethik in der Medizin 23, 303-314

Mayring P (2008) Qualitative Inhaltsanalyse. Grundlagen und Techniken. Beltz Verlag Weinheim, Basel

Strech D, Börchers K, Freyer D, Neumann A, Wasem J, Marckmann G (2008) Ärztliches Handeln bei Mittelknappheit. Ergebnisse einer qualitativen Interviewstudie. Ethik in der Medizin 20, 94-109

Strech D, Danis M (2014) How can bedside rationing be justified despite coexisting inefficiency? The need for 'benchmarks of efficiency'. I Med Ethics 40, 89-93

Strech D, Follmann M, Klemperer D, Lelgemann M, Ollenschlager G, Raspe H, Nothacker M (2014) When Choosing Wisely meets clinical practice guidelines. Z Evid Fortbild Qual Gesundhwes 108, 601-3 